\title{
A Fuzzy Logic Module to Estimate a Driver's Fuel Consumption for Reality-Enhanced Serious Games
}

\author{
Rana Massoud $^{1,2}$, Stefan Poslad $^{2}$, Francesco Bellotti ${ }^{1}$, Riccardo Berta ${ }^{1}$, \\ Kamyar Mehran ${ }^{3}$, Alessandro De Gloria ${ }^{1}$ \\ ${ }^{1}$ University of Genoa Elios Lab, \{rana.massoud, franz, berta\}@elios.unige.it, \\ alessandro.degloria@unige.it \\ ${ }^{2}$ Queen Mary University of London IoT2US Lab, \{r.massoud, stefan.poslad\}@qmul.ac.uk \\ ${ }^{3}$ Queen Mary University of London RPCS Lab, k.mehran@qmul.ac.uk
}

\begin{abstract}
Reality-enhanced gaming is an emerging serious game genre, that could contextualize a game within its real instruction-target environment. A key module for such games is the evaluator, that senses a user performance and provides consequent input to the game. In this project, we have explored an application in the automotive field, estimating driver performance in terms of fuel consumption, based on three key vehicular signals, that are directly controllable by the driver: throttle position sensor (TPS), engine rotation speed (RPM) and car speed. We focused on Fuzzy Logic, given its ability to embody expert knowledge and deal with incomplete information availability. The fuzzy models - that we iteratively defined based on literature expertise and data analysis - can be easily plugged into a reality-enhanced gaming architecture. We studied four models with all the possible combinations of the chosen variables (TPS and RPM; RPM and speed; TPS and speed; TPS, speed and RPM). Input data were taken from the enviroCar database, and our fuel consumption predictions compared with their estimated values. Results indicate that the model with the three inputs outperforms the other models giving a higher coefficient of determination $\left(R^{2}\right)$, and lower error. Our study also shows that RPM is the most important fuel consumption predictor, followed by TPS and speed.
\end{abstract}

Keywords: Serious game, Reality-enhanced games, Fuel consumption, Fuzzy Logic, Driving behaviour, Driving feedback, On-Board Diagnostics-II (OBD-II), Open data, eco-driving;

\section{Introduction}

Transportation is a significant source of pollution [1]. Decreasing fuel consumption (FC) would save money and energy. As driving style is a controllable factor for FC [2], serious games (SGs) - games with a purpose different from pure entertainment - have been developed in order to improve the driving behaviour (e.g., [3][4][5]). High quality SGs are typically designed to transfer knowledge and skills, from game-play to real life [6][7]. This might be achieved by inserting game elements in real-world processes (i.e., through gamification [8][9]), applying Game Theory [10] or in "reality-enhanced" gaming approach - a user's real-world activities do feed a digital game [6][11][12]. Such "reality-enhanced" games (or, more specifically, SGs) are specialization of pervasive games [13][14][15], a game genre in which players are immersed in real-life situations and leverage new types of 
contextual interactions therein (allowing user interaction with a virtual environment) [16]. For "reality-enhanced" games to improve a driver behaviour, a major question concerns how to measure the driver performance, which could then be fed into the digital game in different compelling ways [17].

The goal of this paper is to propose and assess a methodology through which to process vehicular data in real-time, so to compute driver performance assessment values (specifically, on driving efficiency - the less the fuel consumed, the more the driving is considered efficient) for use in automotive "reality-enhanced" SGs, in order to improve driver behaviour. The main requirements, that we developed stem from TEAM, a collaborative industrial project that implemented apps, including SGs, for collaborative mobility [17][5]:

1. Real-time driver performance assessment, with a sample frequency in the order of few seconds, and with negligible computation latency (which is compatible with a typical game's timing).

2. Easy access to sensors.

3. Easy to perform profiling and understandable modelling.

4. The possibility of distinguishing between different performance factors, so to give coaching feedback to the driver.

As an indicator of driver performance in this study, we use FC, which is strongly influenced by driving styles [2][18][19], and can be quantified and validated. FC is not directly accessible through the common OBD-II interface. It must be estimated from other available signals. In general, FC estimation is challenging, taking the fact that it is influenced by several factors (Fig. 1) apart from driving patterns (e.g., driving environment, vehicle maintenance) [20][21]. This corresponds to the general case in which a user's performance should be estimated by considering several different factors.

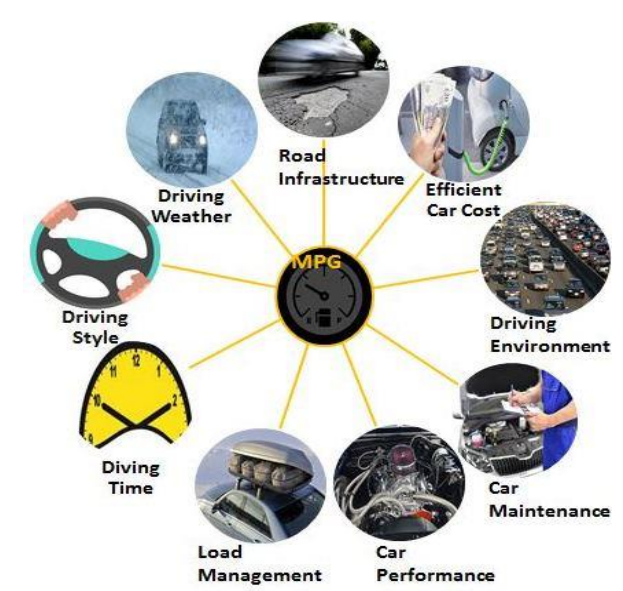

Fig. 1. Influences that affect fuel consumption.

In the light of the above requirements, a Fuzzy Logic (FL) approach was chosen for our project, as a baseline to assess overall feasibility. FL is capable of transferring human knowledge and expertise into a mathematical model by means of if-then rules [22], matching any set of input-output data [23]. Unlike some other data analysis techniques, such as Neural Networks, a FL model itself is readily understandable, which is a key requirement to give coaching feedback to drivers. It uses natural language techniques and variables which are based on the degree of truth, that are easier to understand for humans. FL has been frequently applied to problems with incomplete, imprecise and non-linear data, combining flexibility and simplicity [20][24], and has been considered "promising" in a recent review of driving style analysis systems [25]. Thus, we have first defined a set of vehicular signals that have a significant impact on FC and are controllable by the driver. Then, we developed FL-based estimators, exploiting those sensors on naturalistic driving historical data.

The remainder of the paper is organized as follows: Section 2 reviews the literature; Section 3 presents the data querying process, the feature selection, the modelling process of 
the proposed fuzzy systems; Sections 4 illustrates our experiments to validate and assess the models; conclusions and future works are drawn in Section 5.

\section{State of the Art}

In-car gaming is gaining relevance - it has the potential of making use of all the cool properties of the car itself, the different practices for driving, and driving as a socially shared experience, and challenges (e.g., aspects of driving, such as driving as an arena for gaming) [26]. Several in-car game concept designs have been discussed in [3]. They have also considering the driving style. In [27], an incentive-based mechanism was adopted to improve driver behaviour in managing traffic congestions. [4] evaluated the effects of gamification on driving, especially considering boredom. The Car-wings application by Nissan, represents FC status versus money spent and also provides a comparison among different drivers' performance [28]. The Car2Go application provides gamification features to support environmentally friendly behaviour [29]. [30] discussed the effects of gamifying recreational bicycle riding, with positive and negative consequences. [17] presented the TEAM SG concept architecture, with three layers, distinguishing (1) sensing modules (e.g., the vehicular signals), from (2) game logic (e.g., virtual bank, snake \& ladders, races), from (3) user interface (e.g., on a Smartphone). Decoupling these three sub-systems, allows such an architecture to implement various types of games, fed (e.g., directly as a score or indirectly as an energy factor, or even to use other game mechanics) by the seamless insertion of sensing modules (e.g., to evaluate the drive style, but also other aspects of driver/user performance), and providing different modalities of user interaction.

Driving data analysis is ever more important with the recent advancements in vehicle safety and efficiency; contextual vehicular sensor data analysis, can provide information relevant to driver's style and mobility patterns including understanding traffic patterns [31]. Modern vehicles collect information from hundreds of sensors, that are connected to the Electronic Control Unit (ECU) through a wired sensor network, typically one or more Controller Area Network bus (CAN bus). Some car data can be publicly gained through the On-Board Diagnostics (OBD) universal interface [32]. Increasing studies rely on OBD data to determine driving profiles (e.g., [33][34][35]), to estimate FC (e.g., [36][37][38][39]) and to measure the gas emission of a car (e.g., [36][40]).

FL has been applied in numerous application domains, including driver behaviour analysis. [41] presented a vehicle speed limit model, with a road safety model discussion based on fuzzy rules. A recent review paper has shown the relevance of FL for driving style analysis [25]. FL has been used for driver fatigue and distraction identification (e.g. in [42]), scoring (e.g., in [43][44]), driving style recognition (e.g., in [34][45][46][47][48]) and FC estimation (e.g., in [20][39]). Throttle position sensor (TPS), RPM and car speed - that are our selected sensors - have been used in [39] to develop an estimation system, that outputs a categorical level of FC (very low, low, medium, High, very high).

In eco-drive-oriented Advanced Driving Assistance Systems (ADAS), a model able to predict FC is a prerequisite [49]. Upon [36], the "engine fuel rate" sensor is readable only in relatively very few car models to date as it is not mandatory in the OBD-II standard protocol. Hence, a number of ways have been proposed to estimate FC, e.g., using Mass Air Flow (MAF). Several researchers have studied driver style assessment and categorisation based on FC. A promising and effective approach is to use eco-drive technologies, that monitor the driver behaviour [50][51][52]. Eco-drive has been useful for fuel-efficiency, environmental protection, riding comfort, and noise pollution [35]. Some companies have long since recognized the value of training drivers for this purpose and started providing eco-drive training achieving FC reductions between 2.6\% [53] and 20\% under real-world driving conditions [54]. [55][56] argue that modifying drivers' behaviour through proper feedback can also potentially lead to FC reductions. 
The selected sensors, RPM, TPS and car speed are considered in the five common rules of eco-driving [57][58][59], and they have been used to discriminate between different driving styles [60] and using FL [45][39]. These variables are also easy to access from the car - they are directly available from the OBD-II standard interface.

The standard OBD-II signal list is aimed at vehicular diagnosis. It does not include some other inputs that affect human driving behaviour and hence influence FC such as acceleration and brake pedal pressure. This limitation might be overcome by carefully including synchronized signals from other devices, such as cellular phones, that include inertial sensors (accelerometer, gyroscope, magnetometer [5][61][62]).

\section{Modelling Fuel Consumption}

\subsection{Data querying framework}

As a first choice, we decided to access vehicular information through the standard OBD-II port. An OBD-II interface is legally required in the US since the year of manufacture 1996; in Europe it is legally required for cars with a gasoline engine built from 2001, for diesel vehicles built from 2003 and for trucks built from 2005. In order to have the database to define and measure the accuracy of the model, we relied on the enviroCar platform - a community-based open data collection platform for gathering anonymised vehicular sensor data from naturalistic driving [36]. The community uses standard Bluetooth OBD-II adapters that read vehicular information through the OBD-II port. This information is sampled at regular time intervals. Most of the requested available data tracks are recorded at a $5 \mathrm{~s}$ sampling time by an enviroCar Android Smartphone app and delivered to a server together with added GPS information. Further information, such as FC and Carbon dioxide $\left(\mathrm{CO}_{2}\right)$ emission, are computed and added on the server. The enviroCar community relies on the "Mass Air Flow" (MAF) sensor, which measures the amount of air that flows into the engine. The engine control unit uses this to determine how much fuel must be flown into the engine cylinders. Consistently, our analysis shows a Pearson Product Moment Correlation (PPMC) value equal to 1 between FC and MAF (Fig. 4). Tests conducted in [63] show that the MAF is a best candidate for estimating FC from OBD data. In this work, however, we focused on sensors that are affected by driver style and are easy to explain to return feedback to the driver.

$$
\begin{aligned}
& \text { Fuel consumption }\left(\frac{\mathrm{g}}{\mathrm{s}}\right)=\frac{\text { fuelweight }}{\mathrm{s}}\left(\frac{\mathrm{g}}{\mathrm{s}}\right)=\frac{M A F\left(\frac{g}{s}\right)}{A F R} \\
& \text { Fuel consumption }\left(\frac{\mathrm{l}}{\mathrm{h}}\right)=\frac{\frac{M A F\left(\frac{g}{s}\right)}{A F R}}{\text { Density of fuel }\left(\frac{g}{l}\right)}\left(\frac{\mathrm{l}}{\mathrm{s}}\right) * 3600\left(\frac{\mathrm{l}}{\mathrm{h}}\right)
\end{aligned}
$$

EnviroCar estimates FC for gasoline vehicles through the formula in eq. (1), described in [64], where MAF is measured in (grams/second), AFR is the Air Fuel Ratio (Mass Ratio of Air to fuel), which is 14.7 for gasoline. MAF over AFR is thus in (grams/second). EnviroCar provides the FC in (litres/h), where gasoline has a 745 (grams/litre) density (see eq. (2)). Fuel combustion is complete when there is a ratio of $14.7 \mathrm{~kg}$ of air per $1 \mathrm{~kg}$ of gasoline [36]. Although the MAF sensor is mandatory in the OBD-II standard, it is not supported by some vehicle types. Thus, the enviroCar community estimates it by utilizing other parameters, namely temperature, air pressure, and engine speed [36]. In this work, the focus is on gasoline engines, as enviroCar's FC estimation provides the best accuracy for a gasoline engine rather than diesel engine [65]. 


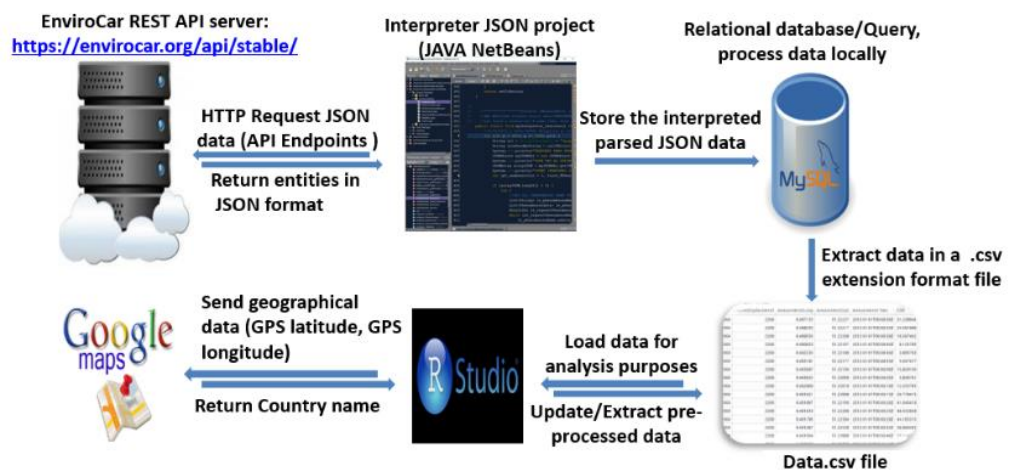

Fig. 2. Data querying system architecture.

The present work leverages the 3 layer TEAM architecture - that can be logically ported to other application domains beyond cars. This can process the signals from the physical sensors on a vehicle to estimate in real-time the driving behaviour of a user that will be employed as a part of a SG. More specifically we address techniques for implementing layer 1 in the TEAM architecture. To access the enviroCar data that is needed to define and validate our FC module for the sensor layer of the TEAM SG concept architecture, we developed a software system which is depicted in Fig. 2.

To do this, we request data through a JSON (JavaScript Object Notation) interface, using enviroCar REST API via HTTP requests [36]. Our system analyses the received data and stores them in a local relational database. In order to recognize the country where the track was recorded, we use "Google Maps API" provided by "ggmap"" library in the process of back (reverse) coding ${ }^{3}$ a point location (latitude, longitude) to a readable address (country, locality, and route). For all our analyses, we considered 1,090 tracks for gasoline engine vehicles, with 733,274 measurements (after ignoring incomplete records), that were recorded mostly in Germany (a few tracks are recorded in other countries e.g., France) in the period 2012-01-01 - 2016-04-19.

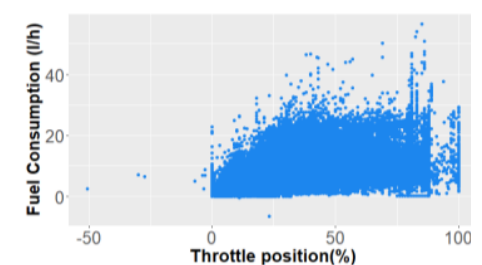

(1)

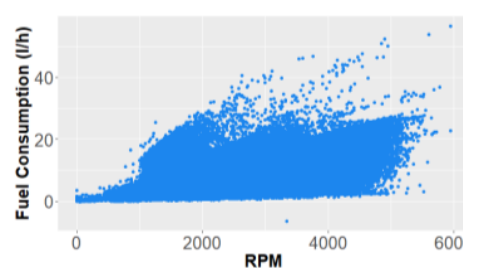

(2)

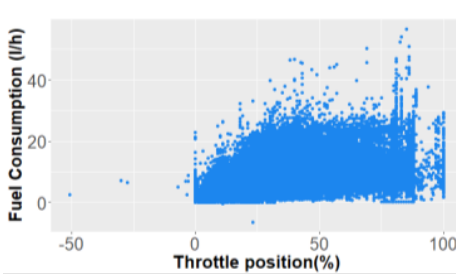

(3)

Fig. 3. Scatterplots from the enviroCar database. (1): FC-TPS.

$$
\text { FC-RPM. (3): FC - Speed. }
$$

As anticipated, we focused on the sensors that determine how driving style impacts the FC in a way that is easily accessible and understandable, to provide direct feedback to the driver. To this end, we analysed the correlation of FC (computed by enviroCar) with the 13 OBDII/enviroCar provided sensors, and finally chose TPS, RPM and car speed. Fig. 3 shows the FC plots against TPS (Fig. 3 (1)), RPM (Fig. 3 (2)), and car speed (Fig. 3 (3)), for all the analysed available data. Fig. 4 shows the correlations

\footnotetext{
${ }^{1}$ Google Maps Platform https://cloud.google.com/maps-platform/

${ }^{2}$ Reverse geocode in $\mathrm{R}$ with ggmap package

https://www.rdocumentation.org/packages/ggmap/versions/2.6.1/topics/revgeocode

${ }^{3}$ Google Maps reverse geocoding

https://developers.google.com/maps/documentation/geocoding/start

International Journal of Serious Games
} 


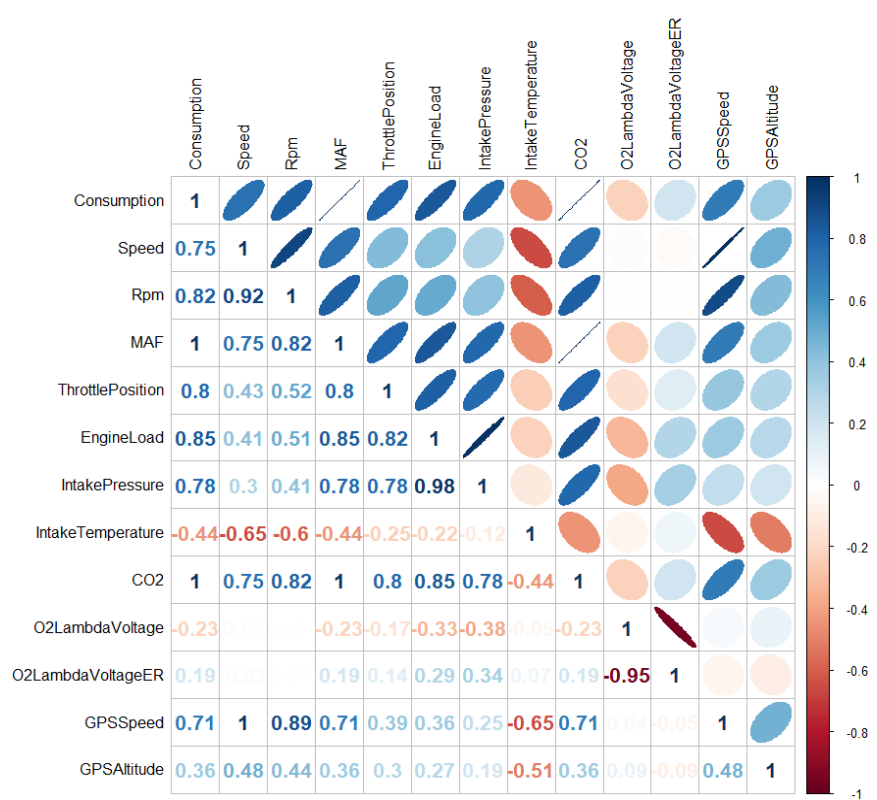

Fig. 4. Correlation between enviroCar estimated FC and car variables [ellipse shapes and colours are explained in the text section 3.1].

in a symmetric matrix between some OBD-II variables in our database and the FC values estimated by the community. The Pearson Product Moment Correlation (PPMC) between FC and our selected variables is quite high $(0.8,0.82$ and 0.75 , for TPS, RPM, and speed respectively). On one side, the matrix shows the explicit values of the correlation: high correlation values between 0.5 to 1 (eclipses tend to more dark blue colour) or -0.5 to -1 (eclipses tend to more dark wine red colour); medium correlation between 0.3 to 0.5 (eclipses tend to medium blue colour) or -0.3 to -0.5 (eclipses tend to light coral colour); low correlation between 0.1 to 0.3 (eclipses tend to light blue colour) or -0.1 to -0.3 (eclipses tend to light coral colour); 0 indicates no correlation between variables (eclipses with transparent colour). Ellipses with a shape close to straight line indicate a pair of tightly linked sensors, that may be directly or inversely correlated, depending on the direction of the ellipse's main axis.

\subsection{The fuzzy model}

The Mamdani FL model [66][67], that we follow in this work, considers linguistic variables (e.g. very cold, slightly hot) in both the antecedent and consequent parts of the rules. So, in multi-input and single-output (MISO) systems, fuzzy IF-THEN rules are of the following form (3):

$$
\text { IF } \mathrm{X} 1 \text { is } A_{1} \text { and } \ldots \text { and } X_{n} \text { is } A_{n} \text { THEN } Y \text { is } B \text {, }
$$

where $\mathrm{X}_{\mathrm{i}}$ is input linguistic variable and $\mathrm{Y}$ is output linguistic variable, and $\mathrm{A}_{\mathrm{i}}$ and $\mathrm{B}$ are linguistic values. The standard workflow for the Mamdani model is displayed in Fig. 5, with 3 main steps:

1. Fuzzification: convert classical (crisp) data into fuzzy data or membership functions (MFs).

2. Inference: combine the fuzzy set definitions and parameters of MFs with the fuzzy control rules applied, which can be considered as the knowledge of an expert in the field of application, to derive the fuzzy output.

3. Defuzzification of the output distribution: convert the linguistic fuzzy output (resulted from the previous step) back to the classical output (real value). 


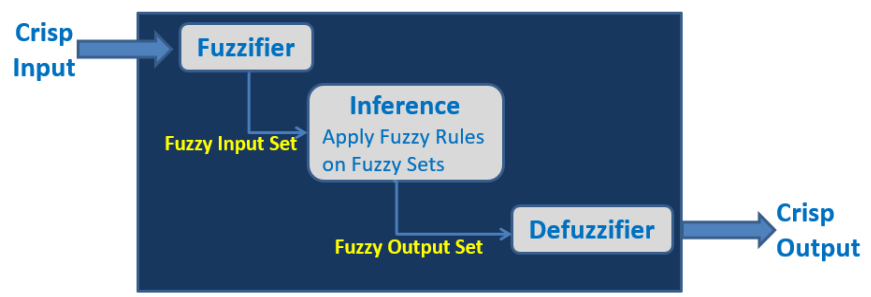

Fig. 5. Fuzzy Inference System of Mamdani model.

In this work, the fuzzy rules were built based on the fuzzy AND operator and all the rule weights are set equal to 1 . Defuzzification is done based on the centroid technique. The first step of the process consists in specifying the variable ranges. Observing the available data, we consider 0-100 (\%) the domain for the TPS range; 0-6000 for RPM range; 0-200 (km/h) is for car speed range; and 0-30 (1/h) for FC range. In order to identify the best solutions, we tested several alternatives, starting with the definition of MFs of the various variables. For TPS, after several experimental attempts, also with more levels, we employed the MFs determined through expert driver knowledge in [45]. For RPM, car speed and FC, as shown later in this section, we defined, our own MFs based on logic, and then refined them through trial and error, which we employed for the definition of the rules too.

To define the rules, that decide the output MF for each combination of the inputs, we exploit the following statistical information: First quartile $\left(\mathrm{Q}_{1}\right)$, which is the middle number between the smallest number and the median of the data set; Median, that marks the middle of the data in the sense that half of the data is less than the median; and the Third quartile $\left(\mathrm{Q}_{3}\right)$, which is the middle value between the median and the highest value of the data set.

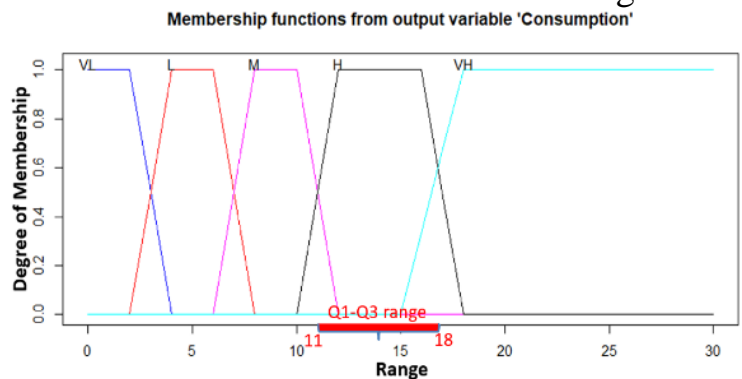

Fig. 6. Example of rule selection.

In the following, we show an example of building a rule for fuzzy inference system 1 (FIS1) with TPS and RPM as inputs, described in the next section [3.2.1. FIS1: TPS and RPM] and its MF plots are illustrated in Fig. 8. Fuzzy values of TPS are defined to be Low $(\mathrm{L})$, Medium (M) and High (H), as specified through expert knowledge in [45]. Five MFs are used for "RPM": Very Low (VL), Low (L), Medium (M), High (H) and Very High (VH). The MFs of the output, are defined to be Very Low (VL), Low (L), Medium (M), High (H) and Very High (VH). To estimate the FC level when (RPM is H) AND (TPS is H), we filter the FC data satisfying the condition: $(2500<\mathrm{RPM}<4000)$ AND (TPS $>60)$ and get the following statistical information: $\mathrm{Q}_{1}=11.76, \mathrm{Q}_{3}=18$ and Median $=14.79$. Considering our FC MFs ( $\{$ Very Low (VL), Low (L), Medium (M), High (H), Very High (VH) $\}$ ) (Table 3 and Fig. 8 (c)), the $\mathrm{Q}_{1}-\mathrm{Q}_{3}$ range overlaps with three FC MFs: M, H and VH), with most of the data is in the $\mathrm{H}$ level (Fig. 6). The median is in $\mathrm{H}$ as well. We thus deduce the rule: IF (RPM is H) AND (TPS is H) THEN FC is $\mathrm{H}$. If the median and $\mathrm{Q}_{1}-\mathrm{Q}_{3}$ criteria do not agree (e.g., the median might be in the intersection of two different FC levels), some more processing would be needed. These uncertain cases are handled by measuring the performance of the fuzzy estimator in the two possible output levels and deciding the level that returns the smaller Mean-squared-error (MSE) with respect to the reference value. The overall process is illustrated in Fig. 7. 


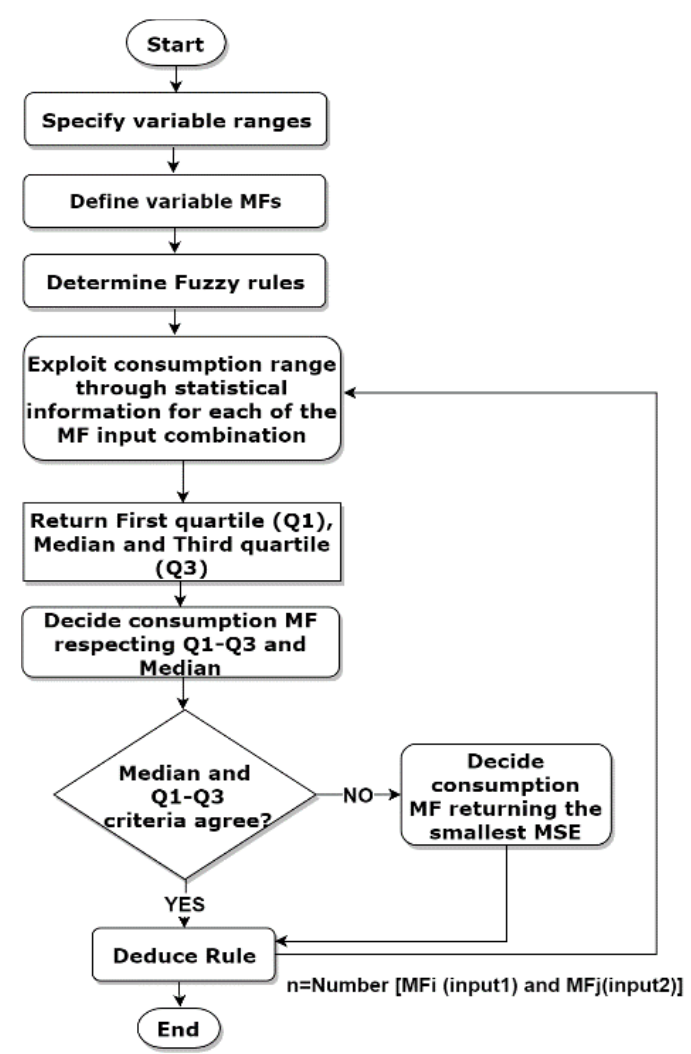

Fig. 7. The fuel consumption algorithm.

In the following text, we present our best performing implementations of FL-based modules for the four possible combinations of the selected input sensors, namely FIS1, with TPS and RPM; FIS2, with car speed and RPM; FIS3 with TPS and car speed; and FIS4 with all the three inputs.

\subsubsection{FIS1: TPS and RPM}

FIS1 includes two inputs: TPS and RPM. The MFs of two variables with their ranges are shown in Table 1 and Table 2 respectively.

Table 1. Membership Functions of TPS (\%) input.

\begin{tabular}{|c|c|c|c|}
\hline Membership Function & L & M & H \\
\hline Throttle Position (\%) & $0-40$ & $20-80$ & $60-100$ \\
\hline
\end{tabular}

Table 2. Membership Functions of RPM input.

\begin{tabular}{|c|c|c|c|c|c|}
\hline Membership Function & VL & L & M & H & VH \\
\hline RPM & $0-1000$ & $500-2000$ & $1500-3000$ & $2500-4000$ & $3500-6000$ \\
\hline
\end{tabular}

The fuzzification step of the two inputs, is made by their MFs. The two MF plots are shown in Fig. 8 (1) and Fig. 8 (2). The fuzzy controller output is derived from fuzzification of both inputs and outputs, defining the associated MF. The MFs of the FC are shown in Table 3, and the corresponding MF plots are presented in Fig. 8 (3).

Table 3. Membership Functions of FC (l/h) output.

\begin{tabular}{|l|c|c|c|c|c|}
\hline Membership Function & VL & L & M & H & VH \\
\hline Fuel consumption (1/h) & $0-4$ & $2-8$ & $6-12$ & $10-18$ & $15-30$ \\
\hline
\end{tabular}




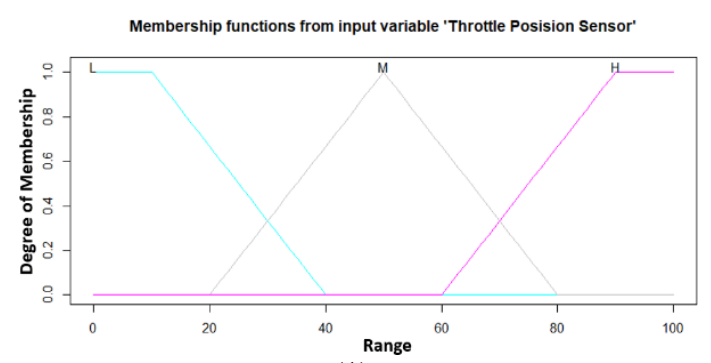

(1)

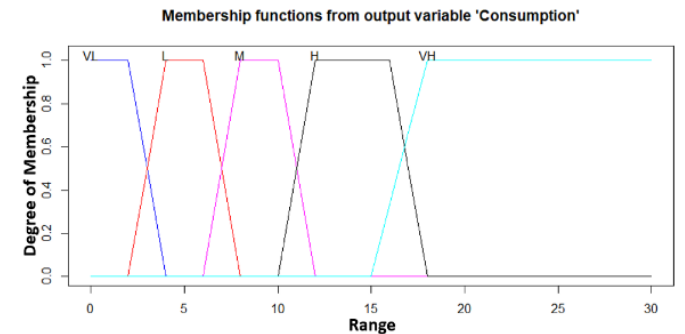

(3)

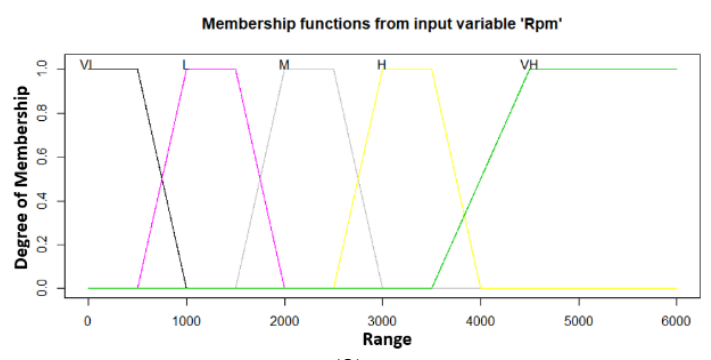

(2)

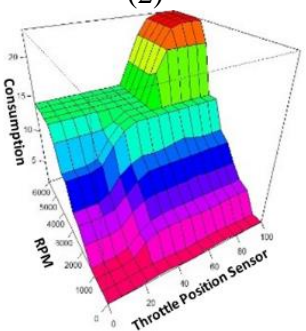

(4)

Fig. 8. MFs of FIS1 variables. (1): TPS MFs. (2): RPM MFs. (3): MFs of FC output. (4): Whole process mapping of FIS1 by output surface plot for TPS, RPM, and FC.

Table 4. Fuzzy Rules matrix for FIS1.

\begin{tabular}{|c|c|c|c|c|c|}
\hline $\begin{array}{c}\text { RPM } \\
\text { TPS }\end{array}$ & VL & L & M & H & VH \\
\hline $\mathbf{L}$ & VL & VL & L & M & H \\
\hline M & VL & L & M & H & H \\
\hline H & VL & L & M & H & VH \\
\hline
\end{tabular}

The fuzzy rules with the AND operator are provided in Table 4. An example of rule is thus the following: when TPS is ' $\mathrm{M}$ ' and RPM is 'VH', FC is ' $\mathrm{H}$ '. The FIS1 three-dimensional view on the data - output surface plot representing the dependency of the FC output on the two inputs - is shown in Fig. 8 (4). It is a three-dimensional surface that represents the mapping from TPS and RPM to FC.

\subsubsection{FIS2: Car speed and RPM}

The second combination involves the car speed and RPM sensors. The MFs of car speed with its ranges, are shown in Table 5. Corresponding fuzzy values are defined to be Low (L), Medium (M), High (H) and Very High (VH). The plots of MFs for the inputs and output (FC) are shown in Fig. 9 respectively: (1) Car speed MFs plot; (2) RPM MFs plot; (3) FC MFs plot. Fig. 9 (4) displays the control surface generated by the fuzzy system; FC output is plotted against the two input variables (car speed and RPM).

Table 5. Membership Functions of car speed $(\mathrm{km} / \mathrm{h})$ input.

\begin{tabular}{|c|c|c|c|c|}
\hline Membership Function & L & M & H & VH \\
\hline Car speed (km/h) & $0-60$ & $40-100$ & $80-140$ & $110-200$ \\
\hline
\end{tabular}




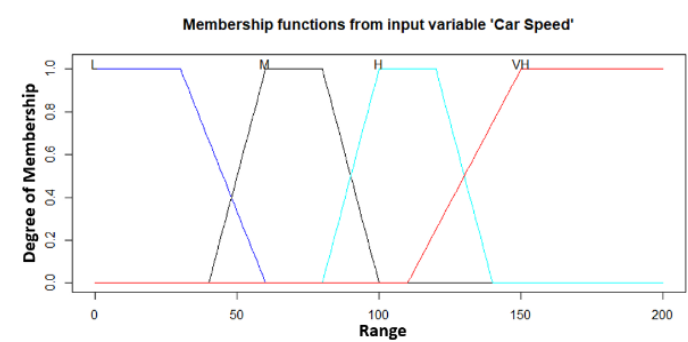

(1)

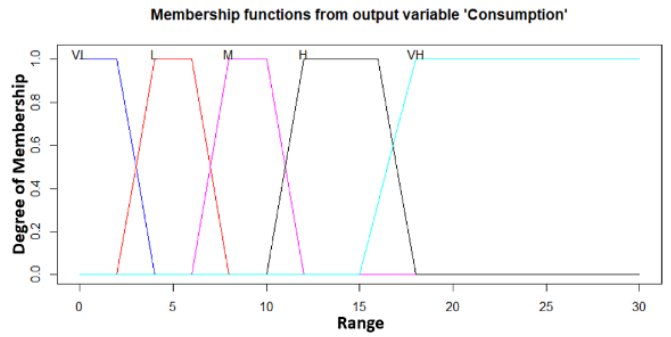

(3)

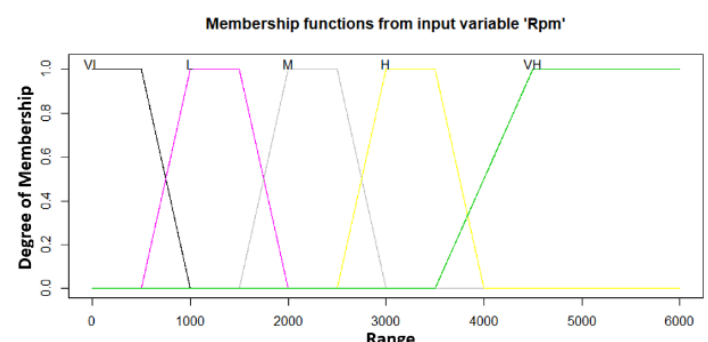

(2)

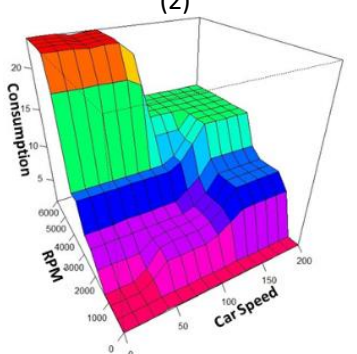

(4)

Fig. 9. MFs of FIS2 variables. (1): Car speed MFs. (2): RPM MFs. (3): MFs of FC output. (4): Whole process mapping of FIS2 by output surface plot for speed, RPM, and FC.

The deduced Fuzzy rules are provided in Table 6. For instance, if car speed is ' $\mathrm{H}$ ' and RPM is ' $\mathrm{H}$ ', $\mathrm{FC}$ is ' $\mathrm{M}$ ' and if the speed is ' $\mathrm{L}$ ' or ' $\mathrm{M}$ ', yet the RPM is ' $\mathrm{VH}$ ', then the $\mathrm{FC}$ is ' $\mathrm{VH}$ ' because the vehicle is being driven less efficiency than if the speed was 'VH' and the RPM is ' $\mathrm{VH}$ '.

Table 6. Fuzzy Rules matrix for FIS2.

\begin{tabular}{|c|c|c|c|c|c|}
\hline $\begin{array}{r}\text { RPM } \\
\text { Speed }\end{array}$ & VL & L & M & H & VH \\
\hline L & VL & VL & L & M & VH \\
\hline M & VL & L & L & M & VH \\
\hline H & VL & L & L & M & H \\
\hline VH & VL & M & M & H & H \\
\hline
\end{tabular}

\subsubsection{FIS3: Car speed and TPS}

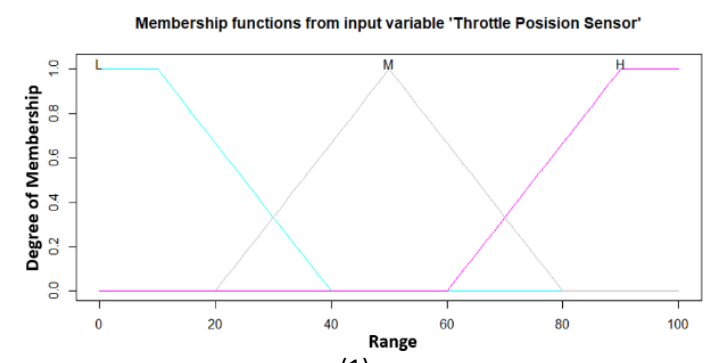

(1)

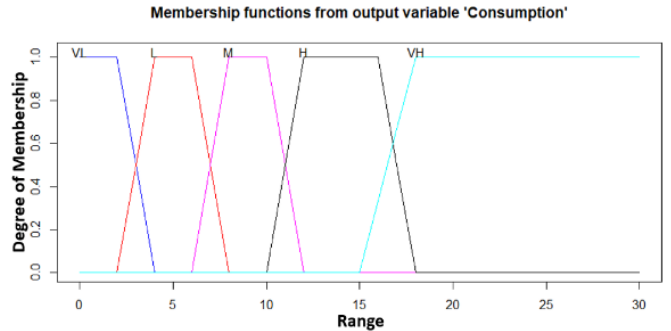

(3)

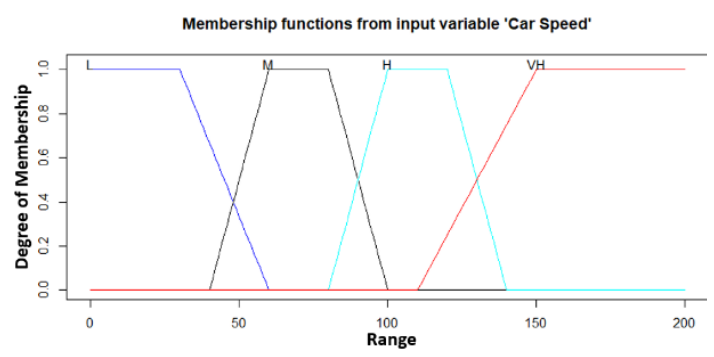

(2)

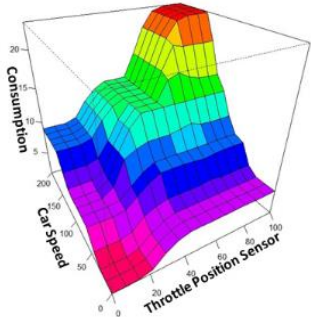

(4) 
Fig. 10. MFs for the inputs and output of FIS3. (1): TPS MFs. (2): Car speed MFs. (3): MFs of FC output. (4): Whole process mapping of FIS3 by output surface plot for TPS, speed, and $F C$.

The combination of TPS and car speed is modelled in FIS3 (Fig. 10). The 3D interpretation of the FIS3 input-output relations, is illustrated with an output surface in Fig. 10 (4).

Table 7. Fuzzy Rules matrix for FIS3.

\begin{tabular}{|c|c|c|c|c|}
\hline $\begin{array}{c}\text { Speed } \\
\text { TPS }\end{array}$ & L & M & H & VH \\
\hline L & VL & L & L & M \\
\hline M & L & M & H & H \\
\hline H & L & M & H & VH \\
\hline
\end{tabular}

\subsubsection{FIS4: Car speed, RPM and TPS}

The last FIS includes all the three inputs, for which the MFs were shown previously. Sixty possible combinations of the variable MFs with AND operator were studied, with TPS ( $\{\mathrm{L}$, $\mathrm{M}, \mathrm{H}\}), \mathrm{RPM}(\{\mathrm{VL}, \mathrm{L}, \mathrm{M}, \mathrm{H}, \mathrm{VH}\})$, and speed ( $\{\mathrm{L}, \mathrm{M}, \mathrm{H}, \mathrm{VH}\})$ respectively. After the synthesis step - deciding, with the suggested approach (Fig. 7), the following seventeen rules have been deduced:

1. if RPM is VL then FC is VL

2. if RPM is $\mathrm{L}$ and TPS is $\mathrm{L}$ and Speed is $(\mathrm{L}$ or $\mathrm{M}$ or $\mathrm{H})$ then $\mathrm{FC}$ is $\mathrm{VL}$

3. if RPM is L and TPS is $\mathrm{L}$ and Speed is VH then FC is $\mathrm{L}$

4. if RPM is $L$ and TPS is $M$ then FC is $M$

5. if RPM is $L$ and TPS is $H$ and Speed is $(L$ or $M)$ then $F C$ is $L$

6. if RPM is L and TPS is $H$ and Speed is $H$ then FC is VH

7. if RPM is $L$ and TPS is $H$ and Speed is VH then FC is $H$

8. if RPM is $M$ and TPS is $L$ then $F C$ is $L$

9. if RPM is $M$ and TPS is $M$ then FC is $M$

10. if RPM is $M$ and TPS is $H$ then $F C$ is $M$

11. if RPM is $H$ and TPS is $L$ then FC is $L$

12. if RPM is $H$ and TPS is $M$ and Speed is $L$ then FC is $M$

13. if RPM is $\mathrm{H}$ and TPS is $\mathrm{M}$ and Speed is (M or $\mathrm{H}$ or $\mathrm{VH}$ ) then $\mathrm{FC}$ is $\mathrm{H}$

14. if RPM is $\mathrm{H}$ and TPS is $\mathrm{H}$ then FC is $\mathrm{H}$

15. if RPM is VH and TPS is $L$ then $F C$ is $M$

16. if RPM is VH and TPS is $M$ then $F C$ is $H$

17. if RPM is VH and TPS is $\mathrm{H}$ then $\mathrm{FC}$ is $\mathrm{VH}$

The reduction to seventeen rules was obtained by synthetizing all the sixty resulting rules. For instance, when RPM is 'VL', FC is always 'VL', independent of the level of TPS and speed. The 12 rules where RPM is 'VL' could thus be summarised by the first rule above (if RPM is VL then FC is VL). In general, the rules highlight a higher impact on FC of RPM, compared to speed and TPS. We also argue that TPS is the second informative ones for the model. As an example, in rules (15)-(17), if we have "Very High" level for RPM, the output is based on the TPS value: "Low" TPS implies "Medium" FC; "Medium" TPS implies "High" FC; "High" TPS implies "Very High" FC.

\section{Experimental Results and Discussion}

The Fuzzy models were developed using the "FuzzyToolkitUoN" R package [68], and the experimental tests were conducted in the same computational environment, on an 8 GB RAM and i7-770 CPU desktop PC. For each one of our four FL models, every input sample was 
processed within 0.01 seconds (this is thus our estimator's latency time experienced by a user). FC output values of our fuzzy inference systems (FISs) were then compared with the consumption reported in the enviroCar database.

Table 8. Model performance comparison.

\begin{tabular}{|c|c|c|c|c|c|c|c|c|}
\hline $7^{\text {Model }}$ & TPS & $\begin{array}{l}\text { S1 } \\
\text { RPM }\end{array}$ & $\begin{array}{r}\mathbf{F} \\
\text { Speec }\end{array}$ & RPM & $\begin{array}{l}1 \\
\text { TPS } \\
\end{array}$ & $\begin{array}{l}3 \\
3 \\
\text { Speed }\end{array}$ & $\begin{array}{r}\mathbf{F} \\
\text { three }\end{array}$ & $\begin{array}{l}4 \\
\text { puts }\end{array}$ \\
\hline Trac & $\mathbf{R}^{2}$ & MSE & $\mathbf{R}^{2}$ & MSE & $\mathbf{R}^{2}$ & MSE & $\mathbf{R}^{2}$ & MSE \\
\hline All available gasoline & 0.62 & 5.8 & 0.47 & 8.25 & 0.64 & 5.52 & 0.71 & 4.69 \\
\hline Same car & 0.85 & 1.75 & 0.53 & 4.75 & 0.76 & 3.53 & 0.86 & 1.52 \\
\hline
\end{tabular}

Table 8 provides the differences between the developed models outcome and the target FC provided by the enviroCar database in terms of $\mathrm{R}$ squared $\left(\mathrm{R}^{2}\right)$ and the Mean-Squared-Error (MSE). The model with three inputs (FIS4) is the best one, with the highest $\mathrm{R}^{2}(0.71)$ and the lowest MSE (4.69). FIS1 and FIS3 return good results, with $\mathrm{R}^{2}$ equal to 0.62 and 0.64 , respectively, and with MSE equal to 5.8 and 5.52, respectively. FIS2 is not as accurate as the other models, achieving the lowest $\mathrm{R}^{2}(0.47)$ and the highest MSE (8.26). The absence of TPS in FIS2 suggests its importance. A parametric analysis would clarify and validate our assumption by investigating how each of the considered variables actually influences FC (e.g. [69]).

In order to check the effect of some of the other factors illustrated in Fig. 1, and not captured by our sensors, we evaluated the models on data from the same car. We thus considered 111 different driving tracks with 47,076 measurements (after ignoring incomplete records) for a gasoline car with the following characteristics: Volkswagen (VW) manufacturer, $9 \mathrm{~N}$ model constructed in 2009. Results in Table 8, show a higher accuracy than in the general case (when working on different car types), for all the four models, with higher $\mathrm{R}^{2}$ values $0.85,0.53,0.76$ and 0.86 and lower MSE values $1.75,4.75,3.53$, and 1.52 for FIS1, FIS2, FIS3 and FI4 respectively. This result suggests the importance of also considering other factors (e.g. car characteristics such as engine size, number of cylinders, engine displacement) in estimating FC.

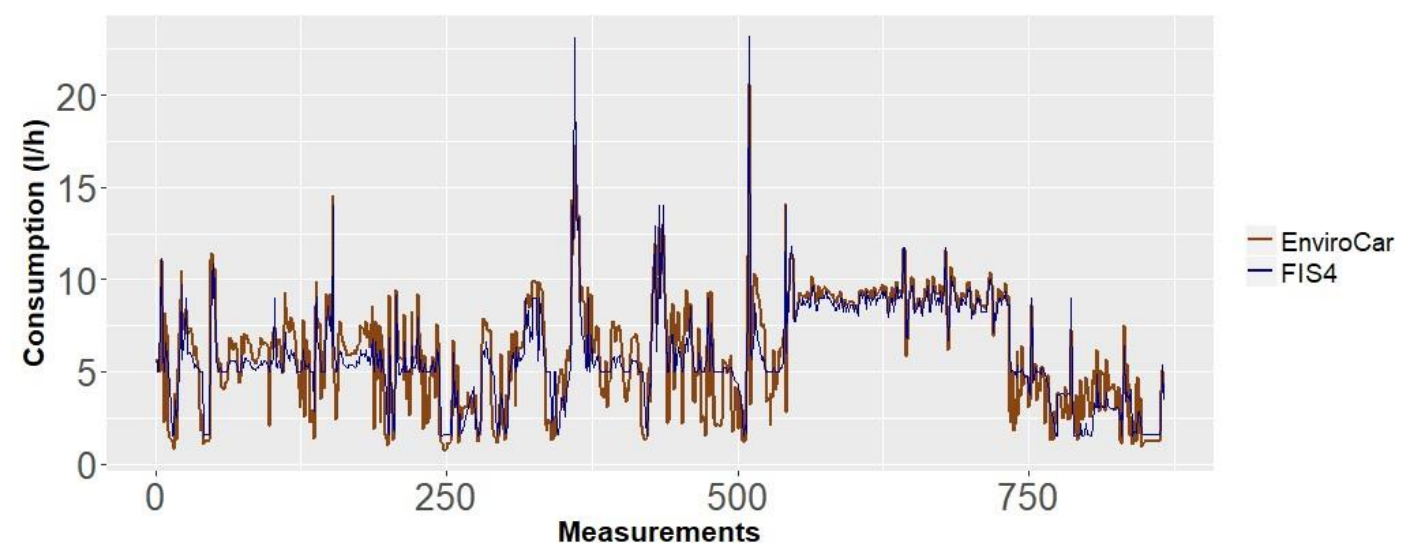

Fig. 11. Evaluation of FIS4 with a track.

Fig. 11 shows the time evolution of our estimation (obtained with the FIS4 model) vs the actual enviroCar data in a sample track. It is clear that the predictor is able to follow the main peaks of the signal (even without the possibility of having a direct input about acceleration/deceleration), while it has difficulty in capturing small variations around the average value around $5 \mathrm{l} / \mathrm{h}$. We argue that our FL module might be improved in those cases by introducing other input sensors, for instance, the engine load sensor.

As anticipated, one of the expected benefits of employing FL consists of the possibility of providing coaching feedback to the driver (the link between outcomes with inputs) and by the fact that the rules are applied to input values that - at least in our case - can be directly 
controlled by the user. As a simple example, upon the 14th rule of FIS4, if a "High" FC is obtained because of a "High" RPM and a "High" TPS value, we can advise the player to decelerate, or to shift up a gear.

\section{Conclusion and Future Work}

Reality-enhanced gaming is an emerging serious game genre, that looks beneficial particularly because of its ability to contextualise a game within a real target environment. A key module for such games is the evaluator that senses a field user's or worker's performance and provides consequent input to the Serious Game (SG). In this project, we have explored this field, focusing on estimating automotive driver performance in terms of FC, based on TPS, RPM and car speed variables read from the OBD-II interface. In a baseline approach, we have focused on FL, a promising technique for driving style analysis [25], that gives the possibility of distinguishing between different performance factors, so to give coaching feedback to the driver. Thus, a major contribution of this paper consists in showing a process that might be implemented and adapted to other instructional domains as well. Four fuzzy inference systems (FISs) with all the possible combinations of the inputs, have been modelled: FIS1 (TPS and RPM), FIS2 (speed and RPM), FIS3 (TPS and speed) and FIS4 (with all the three inputs). It has been defined on the basis of naturalistic driving data (733,274 measurements) and was not calibrated for a specific car model, which made the work challenging. Data were taken from the enviroCar database, and our FC predictions were compared with theirs, with a regression analysis for a track. The MFs and fuzzy rules for the models have been defined based on literature expertise, data analysis and trial and error. The developed FC models can be easily plugged in a reality-enhanced gaming architecture (e.g. [17]), as the estimated value can then be seamlessly fed in different game logics and treated according to their specificity. Results indicate a $\mathrm{R}^{2}$ best value of 0.71 and 4.69 for MSE, with FIS4 in the regression evaluation process with the observed consumption provided by enviroCar. The deduced fuzzy rules show that RPM is the strongest fuel consumption predictor, followed by TPS and car speed.

Our FIS1 and FIS4 models achieve good results when dealing with only one vehicle, confirming the importance of considering other variables beyond those we got from the OBDII port, such as vehicle characteristics (e.g., engine size and displacement). Finally, our approach achieves a real-time performance $(0.01 \mathrm{~s}$. response time), which is needed for gaming.

These results are promising, and further work is required to improve estimation accuracy. We plan to work on other defuzzification techniques and check how they influence the outcomes. A Machine Learning (ML) approach could be tested to automatically generate the fuzzy rules. More complex models could be designed, including other variables representative of the driving pattern not provided by the OBD-II interface, such as acceleration and deceleration, that could be considered by exploiting inertial sensors available in cellular phones (e.g., [5]). The effect of external influences should be considered as well, such as time of the day, state of the road, and weather.

We also argued that the proposed FL framework can include the indirect uncertainties in measurement and it can make possible to lower the particulate matters (PM) and FC simultaneously using Mass Air Flow (MAF) and in-cylinder pressure. The fact that in addition to FC, the PM has a more important role in the vehicle overall emission comparing to harmful gases Nitrogen Oxide $\left(\mathrm{NO}_{\mathrm{x}}\right)$, Hydrocarbon $(\mathrm{HC})$ and Carbon Monoxide (CO), which by using the three-way catalyst and stoichiometric combustion can be comfortably reduced to the European standards. Measuring the PM concentration levels is directly not feasible. Employing novel Gasoline Direct Injection (GDI) technology in modern engines for this purpose still faces a number of challenges in acquiring accurate PM data. The concentration level, indicated by specific particulate matter by mass (ISPMM) in $\mathrm{g} / \mathrm{kWh}$ (see eq. (4)), is directly related to the measurement of fuel mass flow rate (MFF) and air mass 
flow (MAF) rate in $\mathrm{g} / \mathrm{min}$. CAM refers to concentration accumulation mode weighted by mass and MMEx is the Molar Mass of Emissions [70][71].

$$
I S P M M=\frac{C A M \cdot 10^{-6} \cdot 10^{3} \cdot(M A F+M F F) \cdot 60}{P_{i} \cdot \frac{M M E x}{22.7 L} / \mathrm{mol}}
$$

To lower the PM concentration, it is necessary to normalize the FC and PM emissions values by engine power outputs $\left(P_{i}\right)$, which is, in turn directly related to the in-cylinder pressure measured via the Electronic Control Unit (ECU) in every engine cycle, e.g. 10ms. The proposed FL approach can include such indirect measurement uncertainties in cylinder pressure, MAF, and MFF due to other environmental variables and inherent measurement inaccuracies in current sensor technology. Therefore, it can give more accurate results in reducing both the FC and PM in case of measurement uncertainties, compared to other Artificial Intelligence (AI) or ML approaches, which heavily depend on direct sensory data and cannot cope well with measurement uncertainties.

We will explore a FL model to specifically reduce the PM concentration level which is currently a demanding task due to lack of sensor technology for such direct but critical measurements. In this way, we are interested in understanding the possible benefit of combining data-driven models with our FL model, and ML techniques such as Neural Networks, Random Forests and Support Vector Regression in enhancing the model accuracy and overall integrability in a SG design workflow. Considering other variables (even if not directly controllable by the driver), such as engine load (with PPMC equal to 0.85, Fig. 4), could help in increasing the model accuracy with much more complex model design.

If players are to interact with a Reality-enhanced gaming system, it is paramount that they trust that the system offers sufficient security, including privacy for their driving behaviour information - this trust must be managed by the system [72]. Note many IoT devices even in vehicles may have zero or minimal security by design because of the use of low resource, low power devices or because they are designed to work as closed vertical services. Security threats and risks may be higher because devices are unattended, use local wireless communication that have no or weak encryption making them more susceptible to eavesdropping and because users find the security too unusable to setup and operate and hence leave devices relatively unsecure. For these reasons, the security and privacy support by the system needs to be designed to be adaptive [73].

\section{Acknowledgements}

We acknowledge that this research was partially funded as part of a Joint Doctorate Interactive and Cognitive Environments (JD-ICE) between the University of Genova Dipartimento di Ingegneria navale, elettrica, elettronica e delle telecomunicazioni (DITEN), Elios Lab in agreement with Queen Mary University of London. We also acknowledge technical support given by the enviroCar open Citizen Science Platform for traffic monitoring and environment (in particular Christoph Stasch and Arne de Wall), led by several project partners, including 52north with whom we liaised.

\section{References}

[1] European Environment Agency. Transport indicators tracking process towards environmental targets in Europe. TERM 2011. Retrieved from

https://www.eea.europa.eu/publications/foundations-for-greener-transport/at_download/file on 2018-12-11. 
[2] Jack N., Barkenbus. Eco-driving: An overlooked climate change initiative. Energy Policy, 38(2):762-769, 2010. https://doi.org/10.1016/j.enpol.2009.10.021

[3] Sven Krome, Steffen P.,Walz, Stefan Greuter, Jussi Holopainen, Ansgar Gerlicher, and Markus Schleehauf. Exploring game ideas for stresslessness in the automotive domain. In Proceedings of the 2014 Conference on Interactive Entertainment, pages 1-3, 2014.

[4] Fabius Steinberger, Ronald Schroeter, and Christopher N Watling. From road distraction to safe driving: Evaluating the effects of boredom and gamification on driving behaviour, physiological arousal, and subjective experience. Computers in Human Behavior, 75:714-726, 2017. https://doi.org/10.1016/j.chb.2017.06.019

[5] Gautam Ravindra Dange, Pratheep Kumar Paranthaman, Francesco Bellotti, Riccardo Berta, Alessandro De Gloria, Mattia Raffero, and Stefan Neumeier. Deployment of serious gaming approach for safe and sustainable mobility. In Intelligent Vehicles Symposium (IV), 2017. https://doi.org/10.1109/IVS.2017.7995879

[6] Jan Dirk Fijnheer and Herre Van Oostendorp. Steps to design a household energy game. In International Conference on Games and Learning Alliance, pages 12-22. Springer, 2015.

[7] Anton Gustafsson, Cecilia Katzeff, and Magnus Bang. Evaluation of a pervasive game for domestic energy engagement among teenagers. Computers in Entertainment (CIE), 7(4):54, 2009. https://doi.org/10.1145/1658866.1658873

[8] Sebastian Deterding, Dan Dixon, Rilla Khaled, and Lennart Nacke. From game design elements to gamefulness: defining gamification. In Proceedings of the 15th international academic Mind Trek conference: Envisioning future media environments, pages 9-15, 2011. https://doi.org/10.1145/2181037.2181040

[9] Stefan Poslad, Athen Ma, Zhenchen Wang, and Haibo Mei. Using a smart city IoT to incentivise and target shifts in mobility behaviour-Is it a piece of pie?. Sensors, 15(6):13069-13096, 2015. https://doi.org/10.3390/s150613069

[10] Haibo Mei, Stefan Poslad, and Shuang Du., A Game-Theory Based Incentive Framework for an Intelligent Traffic System as Part of a Smart City Initiative. Sensors, 17(12):2874, 2017. https://doi.org/10.3390/s17122874

[11] Martijn Kors, Erik D Van der Spek, and Ben AM Schouten. A Foundation for the Persuasive Gameplay Experience. In FDG, 2015.

[12] Richardos Drakoulis, Francesco Bellotti, Ioannis Bakas, Riccardo Berta, Pratheep Kumar Paranthaman, Gautam Ravindra Dange, Panagiotis Lytrivis, Katia Pagle, Alessandro De Gloria, and Angelos Amditis. A Gamified Flexible Transportation Service for On-Demand Public Transport. IEEE Transactions on Intelligent Transportation Systems, 19(3):921-933, 2018. https://doi.org/10.1109/TITS.2018.2791643

[13] Carsten Magerkurth, Adrian David Cheok, Regan L., Mandryk, and Trond Nilsen. Pervasive games: bringing computer entertainment back to the real world. Computers in Entertainment (CIE3(3):4-4, 2005. https://doi.org/10.1145/1077246.1077257

[14] U., Ritterfeld, Ute Ritterfeld, Michael Cody, and Peter Vorderer. Serious games: Mechanisms and effects. Routledge, 2009. https://doi.org/10.4324/9780203891650

[15] Francesco Bellotti, Riccardo Berta, Alessandro De Gloria, Edmondo Ferretti, and Massimiliano Margarone. Vegame: exploring art and history in Venice. Computer, (9):48-55, 2003. https://doi.org/10.1109/MC.2003.1231194

[16] Inseok Hwang, Youngki Lee, Taiwoo Park, and Junehwa Song. Toward a mobile platform for pervasive games. In Proceedings of the first ACM International workshop on Mobile gaming, pages 19-24, 2012. https://doi.org/10.1145/2342480.2342486

[17] Francesco Bellotti, Sven Kopetzki, Riccardo Berta, Pratheep K Paranthaman, Gautam R Dange, Panagiotis Lytrivis, Angelos Amditis et al. TEAM applications for Collaborative Road Mobility. IEEE Transactions on Industrial Informatics, 2018. https://doi.org/10.1109/TII.2018.2850005

[18] Paulo HL., Rettore, Andre B., Campolina, Leandro A., Villas, and Antonio AF., Loureiro. A method of eco-driving based on intra-vehicular sensor data. In 2017 IEEE Symposium on Computers and Communications (ISCC), pages1122-1127, 2017.

[19] Rosolino Vaiana, Teresa Iuele, Vittorio Astarita, Maria Vittoria Caruso, Antonio Tassitani, Claudio Zaffino, and Vincenzo Pasquale Giofre. Driving behavior and traffic safety: an acceleration-based safety evaluation procedure for smartphones. Modern Applied Science, 8(1):88, 2014. https://doi.org/10.5539/mas.v8n1p88

[20]Faran Baig, Muhammad Waseem Ashraf, Muhammad Imran, Zahoor Ahmed, Muhammad Saleem Khan, and Muhammad Awais Farooq. Fuzzy Logic based Fuel Consumption System. Bahria University Journal of Information \& Communication Technology, 5(1):31, 2012. 
[21] Joao C Ferreira, Jose de Almeida, and Alberto Rodrigues da Silva. The Impact of Driving Styles on Fuel Consumption: A Data-Warehouse-and-Data-Mining-Based Discovery Process. IEEE Trans. Intelligent Transportation Systems, 16(5):2653-2662, 2015. https://doi.org/10.1109/TITS.2015.2414663

[22] Henry Nasution, Hishamuddin Jamaluddin, and Jamaluddin Mohd Syeriff. Energy analysis for air conditioning system using fuzzy logic controller. TELKOMNIKA (Telecommunication $\begin{array}{lllll}\text { Computing Electronics and } & \end{array}$ https://doi.org/10.12928/telkomnika.v9i1.680

[23] Mcniel, D., Freiberger, P., Fuzzy Logic and its uses, SURPRIZE 96, Article 2, "Fuzzy Logic".

[24]Bai, Ying, and Dali Wang. Fundamentals of fuzzy logic control-fuzzy sets, fuzzy rules and defuzzifications. In Advanced Fuzzy Logic Technologies in Industrial Applications, 2006, pp. 1736.

[25] Gys Albertus Marthinus Meiring and Hermanus Carel Myburgh. A review of intelligent driving style analysis systems and related artificial intelligence algorithms. Sensors, 15(12):3065330682, 2015. https://doi.org/10.3390/s151229822

[26] Petra Sundström, David Wilfinger, Alexander Meschtscherjakov, Manfred Tscheligi, Albrecht Schmidt, and Oskar Juhlin. The car as an arena for gaming. In Proceedings of the 14th international conference on Human-computer interaction with mobile devices and services companion, pages 233-236, 2012. https://doi.org/10.1145/2371664.2371722

[27] Rod McCall and Vincent Koenig. Gaming concepts and incentives to change driver behaviour. In Ad Hoc Networking Workshop (Med-Hoc-Net), 2012 The 11th Annual Mediterranean, pages 146-151, 2012.

[28] M., McFarland. Why driving slowly and responsibly can actually be fun. Washington Post, 2014. Retrieved from https://www.washingtonpost.com/news/innovations/wp/2014/06/20/whydriving-slowly-and-0responsibly-can-actually-be-

fun/?noredirect=on\&utm_term=.e25fb875d864 on 2018-06-23

[29] J.,Goldman. Cargo2go's Ecoscore and the Gamification. N.p. Retrieved from https://jaygoldman.com/car2go-s-ecoscore-and-the-gamification-of-driving-d6415ea1045e on 2017-01-17.

[30] Paul Barratt. Healthy competition: A qualitative study investigating persuasive technologies and the gamification of cycling. Health \& place, 46:328-336, 2017. https://doi.org/10.1016/j.healthplace.2016.09.009

[31] Paulo HL, Rettore, Andre B., Campolina, Leandro A., Villas, and Antonio AFLoureiro. Identifying relationships in vehicular sensor data: A case study and characterization. In Proceedings of the 6th ACM Symposium on Development and Analysis of Intelligent Vehicular Networks and Applications, pages 33-40, 2016.

[32] Sunitha Godavarty, Sam Broyles, and Micheal Parten. Interfacing to the on-board diagnostic system. In Vehicular Technology Conference. IEEE-VTS Fall VTC 2000. 52nd, volume 4, 2000. https://doi.org/10.1109/VETECF.2000.886162

[33] Bruce Wallace, Mike Rockwood, Rafik Goubran, Frank Knoefel, Shawn Marshall, and Michelle Porter. Measurement of vehicle acceleration in studies of older drivers from GPS position and OBDII velocity sensors. In Medical Measurements and Applications (MeMeA), 2015 IEEE $\begin{array}{lllll}\text { International Symposium } & \text { on, }\end{array}$ https://doi.org/10.1109/MeMeA.2015.7145234

[34] Oussama Derbel and Rene Jr Landry. Driving style assessment based on the GPS data and fuzzy inference systems. In Systems, Signals \& Devices (SSD), 2015 12th International MultiConference on, pages 1-8, 2015.

[35] Julien Monteil, Niall OHara, Vinny Cahill, and Melanie Bouroche. Real-time estimation of drivers' behaviour. In Intelligent Transportation Systems (ITSC), 2015 IEEE 18th International Conference on, pages 2046-2052, 2015.

[36] Arne Bröring, Albert Remke, Christoph Stasch, Christian Autermann, Matthes Rieke, and Jakob Möllers. enviroCar: A Citizen Science Platform for Analyzing and Mapping Crowd-Sourced Car Sensor Data. Transactions in GIS, 19(3):362-376, 2015. https://doi.org/10.1111/tgis.12155

[37] Chris M Atkinson, Theresa W., Long, and Emil L, Hanzevack. Virtual sensing: a neural networkbased intelligent performance and emissions prediction system for on-board diagnostics and engine control. Technical report, SAE Technical Paper, 1998. Retrieved from https://www.sae.org/publications/technical-papers/content/980516/ on 2018-12-11.

[38] Latha P.S, Menaga N, Manjunath T. K., Efficient Estimation of Fuel Consumption Using Fuzzy Logic. International Journal of Engineering Trends and Technology (IJETT), Vol.16, Oct 2014. 
[39] Avner Pereira, Marcel Alves, and Hendrik Macedo. Vehicle driving analysis in regards to fuel consumption using Fuzzy Logic and OBD-II devices. In Telematics and Information Systems (EATIS), 2016 8th Euro American Conference on, pages 1-4, 2016. https://doi.org/10.1109/EATIS.2016.7520160

[40] Hussam Achour, JG., Carton, and Abdul Ghani Olabi. Estimating vehicle emissions from road transport, case study: Dublin City. Applied energy,88(5):1957-1964, 2011. https://doi.org/10.1016/j.apenergy.2010.12.032

[41] Jafar Pouramini and Ahmad Saeedi. Fuzzy model identification for intelligent control of a vehicle speed limit. Journal of Mathematics and Computer Science, 2:337-347, 2011. https://doi.org/10.22436/jmcs.002.02.14

[42] Fabio Tango and Marco Botta. Real-time detection system of driver distraction using machine learning. IEEE Transactions on Intelligent Transportation Systems, 14(2):894-905, 2013. https://doi.org/10.1109/TITS.2013.2247760

[43] Victor Corcoba Magaña and Mario Muñoz Organero. The impact of using gamification on the eco-driving learning. In Ambient Intelligence-Software and Applications, pages 45-52. Springer, 2014.

[44] German Castignani, Thierry Derrmann, Raphael Frank, and Thomas Engel. Driver behavior profiling using smartphones: A low-cost platform for driver monitoring. IEEE Intelligent

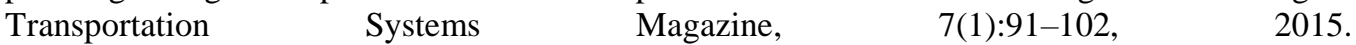
https://doi.org/10.1109/MITS.2014.2328673

[45] Wenshuo Wang, Junqiang Xi, and Xiaohan Li., Statistical pattern recognition for driving styles based on Bayesian probability and kernel density estimation. arXiv preprint arXiv:1606.01284, 2016.

[46] Ahmad Aljaafreh, Nabeel Alshabatat, and Munaf ., Najim Al-Din. Driving style recognition using fuzzy logic. In Vehicular Electronics and Safety (ICVES), 2012 IEEE International Conference on, pages 460-463,2012.

[47] Ashutosh Kumar Choudhary and Piyush K., Ingole. Smart phone based approach to monitor driving behavior and sharing of statistic. In Communication Systems and Network Technologies (CSNT), 2014 Fourth International Conference on, pages 279-282. IEEE, 2014.

[48] Dominik Dorr, David Grabengiesser, and Frank Gauterin. Optimization of system parameters for an online driving style recognition. In Intelligent Transportation Systems (ITSC), 2014 IEEE 17th International Conference on, pages 1021-1026, 2014.

[49] Gennaro Nicola Bifulco, Francesco Galante, Luigi Pariota, and Maria RussoSpena. A linear model for the estimation of fuel consumption and the impact evaluation of advanced driving assistance systems. Sustainability,7(10):14326-14343, 2015. https://doi.org/10.3390/su71014326

[50] Jérôme Barbé and Guy Boy. On-board system design to optimize energy management. In Proceedings of the European Annual Conference on Human Decision-Making and Manual Control (EAM 2006), Valenciennes, France, pages 27-29, 2006.

[51] Olavi H., Koskinen. Improving vehicle fuel economy and reducing emissions by driving technique. In 15th World Congress on Intelligent Transport Systems and ITSAmerica's 2008 Annual MeetingITS AmericaERTICOITS JapanTransCore, 2008.

[52] Michael Sivak and Brandon Schoettle. Eco-driving: Strategic, tactical, and operational decisions of the driver that influence vehicle fuel economy. Transport Policy, 22:96-99, 2012. https://doi.org/10.1016/j.tranpol.2012.05.010

[53] Leverson Boodlal and Kun-Hung Chiang. Study of the Impact of a Telematics System on Safe and Fuel-efficient Driving in Trucks. Technical report, 2014. Retrieved from https://trid.trb.org/view.aspx?id=1312403 on 2018-12-11

[54] Habib Chaari, Eric Ballot, et al. Fuel consumption assessment in delivery tours to develop eco driving behaviour. In European Transport Conference, 2012.

[55] Narelle Haworth and Mark Symmons. Driving to reduce fuel consumption and improve road safety. Monash University Accident Research Centre. http://www.rs conference. com/pdf/RS010036. pdf, 2001.

[56] Mark S., Young, Stewart A., Birrell, and Neville A., Stanton. Safe driving in a green world: A review of driver performance benchmarks and technologies to support 'smart'driving. Applied ergonomics, 42(4):533-539, 2011. https://doi.org/10.1016/j.apergo.2010.08.012

[57] R., Luther and Peter Baas. Eco-driving scoping study, 2011.

[58] The International international situation for promoting eco-driving. Taiwan, 2011.

[59] Bart Beusen, Steven Broekx, Tobias Denys, Carolien Beckx, Bart Degraeuwe, Maarten Gijsbers, Kristof Scheepers, Leen Govaerts, Rudi Torfs, and Luc Int Panis. Using on-board logging devices 
to study the longer-term impact of an eco-driving course. Transportation research part D: transport and environment, 14(7):514-520, 2009. https://doi.org/10.1016/j.trd.2009.05.009

[60] Xiaohua Zhao, Yiping Wu, Jian Rong, and Yunlong Zhang. Development of a driving simulator based eco-driving support system. Transportation Research Part C: Emerging Technologies, 58:631-641, 2015. https://doi.org/10.1016/j.trc.2015.03.030

[61] Thomas Olutoyin Oshin, Stefan Poslad, and Zelun Zhang. Energy-efficient real-time human mobility state classification using smartphones. IEEE Transactions on computers, 64(6):1680$1693,2015$.

[62]Zelun Zhang, Stefan Poslad. Fine-Grained Transportation Mode Recognition Using Mobile Phones and Foot Force Sensors. 9th International Conference on Mobile and Ubiquitous Systems: Computing, Networking and Services (Mobiquitous), Beijing, People's Republic of China, December 12-14, 2012, 103-114. https://doi.org/10.1007/978-3-642-40238-8_9

[63]F., Posada and J., German. Measuring in-use fuel economy in Europe and the US: Summary of pilot studies. Technical report, Working Paper 2013-05). Washington, DC: International Council on Clean ..., 2013. Retrieved from https://www.theicct.org/sites/default/files/publications/ICCT_FuelEcon_pilotstudies_20131125. pdf on 2018-12-11

[64] Asif Faiz, Christopher S Weaver, and Michael P Walsh. Air pollution from motor vehicles: standards and technologies for controlling emissions. The World Bank, 1996. Retrieved from http://siteresources.worldbank.org/INTURBANTRANSPORT/Resources/b02airpolution.pdf on 2018-12-11.

[65] Diesel Engine Fuel Consumption Calculation. Retrieved from https://wiki.52north.org/Projects/DieselConsumptionCalculation on 2018-07-16.

[66] Ebrahim H., Mamdani. Application of fuzzy algorithms for control of simple dynamic plant. In Proceedings of the institution of electrical engineers, volume 121, pages 1585-1588, 1974. https://doi.org/10.1049/piee.1974.0328

[67] Ebrahim H., Mamdani and Sedrak Assilian. An experiment in linguistic synthesis with a fuzzy logic controller. International journal of man-machine studies, 7(1):1-13, 1975. https://doi.org/10.1016/S0020-7373(75)80002-2

[68] Knott, C., Hovell, L., Karimian, N., Garibaldi, J. Package FuzzyToolkitUoN. University of Nottingham IMA Group, 2013. Retrieved from https://cran.rproject.org/web/packages/FuzzyToolkitUoN/FuzzyToolkitUoN.pdf on 2018-12-11.

[69] Eva Ericsson. Independent driving pattern factors and their influence on fuel-use and exhaust emission factors. Transportation Research Part D: Transport and Environment, 6(5):325-345, 2001. https://doi.org/10.1016/S1361-9209(01)00003-7

[70] John B Heywood. Combustion in compression-ignition engines. Internal combustion engine fundamentals, pages 522-562, 1988 .

[71] He Ma. Control oriented engine modelling and engine multi-objective optimal feedback control. $\mathrm{PhD}$ thesis, University of Birmingham, 2013. Retrieved from http://etheses.bham.ac.uk/4308/ on 2018-12-11.

[72] Christian Damsgaard Jensen, Stefan Poslad, Theodosis Dimitrakos. (Eds). Proc. 2nd Int. Conf. on Trust Management, iTrust, Lecture Notes in Computer Science (LNCS), vol. 2995, SpringerVerlag, 2004.

[73] Stefan Poslad, Mohamed Hamdi, and Habtamu Abie. Adaptive security and privacy management for the internet of things (ASPI 2013). In Proceedings of the 2013 ACM conference on Pervasive and ubiquitous computing adjunct publication, pages 373-378, 2013. https://doi.org/10.1145/2494091.2499770 